The Astrophysical Journal, 475:106-117, 1997 January 20

(C) 1997. The American Astronomical Society. All rights reserved. Printed in U.S.A.

\title{
BROAD EMISSION-LINE VARIABILITY IN MARKARIAN 335
}

\author{
Tina M. Kassebaum, Bradley M. Peterson, Ignaz Wanders, Richard W. Pogge, \\ RAY BERTRAM, ${ }^{1}$ AND R. MARK WAGNER ${ }^{1}$ \\ Department of Astronomy, Ohio State University, 174 West 18th Avenue, Columbus, OH 43210-1106 \\ Received 1996 May 10; accepted 1996 August 16
}

\begin{abstract}
We present the results of 6 years of spectroscopic monitoring of the Seyfert 1 galaxy Mrk 335. Each year, the observations span 5-7 months at a nominal sampling rate of one observation per week. Analysis of the spectroscopic observations shows clear coordinated variability in the continuum and in the broad $\mathrm{H} \beta$ and $\mathrm{He}$ II $\lambda 4686$ emission lines. The principal goal of this monitoring program is to measure the time delay between the continuum variations and the corresponding responses of the broad $\mathrm{H} \beta$ and $\mathrm{He}$ II emission lines. We derive time delays of $\sim 17$ days for $\mathrm{H} \beta$ and $\sim 2$ days for He II. From the spectral time series we construct average, root mean square, and difference spectra, which show that the broad $\mathrm{H} \beta$ variations are confined to the core of the broad emission lines, whereas the He II variations take place across the full width of the profile. The He II emission line is about twice as broad as the $\mathrm{H} \beta$ line. Comparison of the $\mathrm{He}$ II and $\mathrm{H} \beta$ lags and line widths indicates that the BLR has a radially stratified ionization structure and that the highest velocity gas is closest to the continuum source. We also find no evidence for correlation between the line profile variations and the continuum and emissionline flux variations, as has been found in other cases. Rather, the line profile variations seem to imply dynamical changes in the BLR.
\end{abstract}

Subject headings: galaxies: active — galaxies: individual (Markarian 335) — galaxies: Seyfert

\section{INTRODUCTION}

Variability of the continuum and emission lines of many active galactic nuclei (AGNs) such as Seyfert 1 galaxies has been recognized as a common property of AGNs for many years (see Peterson 1993 for a recent review). The broad emission lines vary in response to changes in the ionizing flux of the continuum source of the AGN, albeit with a time delay due to light travel time effects in the broad-line region (BLR). Measurements of the time delay between continuum and broad-line variations thus yield information on the spatial scale of the BLR. If emission-line profile variations occur, then these may either signify dynamical changes in the BLR (Perry, van Groningen, \& Wanders 1994) or be a signature of the kinematical structure of the BLR if these profile variations are observed to respond to continuum variations (Blandford \& McKee 1982; Capriotti, Foltz, \& Peterson 1982).

The BLR is a small region $(r \ll 1 \mathrm{pc})$ surrounding a compact $\left(r \lesssim 10^{15} \mathrm{~cm}\right)$ continuum source, which photoionizes the BLR. The standard model (Netzer 1991) is that the BLR consists of a large number of clouds $\left(N_{\mathrm{c}} \gtrsim 10^{6}\right)$ at distances from the continuum source ranging from lightdays $\left(\gtrsim 10^{15} \mathrm{~cm}\right)$ to light-weeks $\left(\gtrsim^{16} \mathrm{~cm}\right)$ for lowluminosity Seyfert 1 galaxies, and larger for higher luminosity sources $\left(r \propto L^{1 / 2}\right.$; Davidson \& Netzer 1979). The $\mathrm{H} \beta$ and $\mathrm{He}$ II emission lines are produced by recombination after photoionization by the continuum source. Hence, the emission-line fluxes vary, with a time delay, along with changes in the ionizing flux from the continuum source. Different emission lines are found to have different response times or lags; the trend is for higher ionization lines to respond more rapidly to the changes in the continuum flux than lower ionization lines (Clavel et al. 1991; Peterson

\footnotetext{
${ }^{1}$ Postal address: Lowell Observatory, Mars Hill Road, 1400 West, Flagstaff, AZ 86001.
}

1993). This trend provides evidence that ionization stratification exists within the BLR.

The study of the size, structure, and kinematics of the BLR through emission-line responses to the continuum variations is known as "reverberation mapping." It is usually assumed that the emission-line light curve $L(t)$ is related to the continuum light curve $C(t)$ through the "transfer equation"

$$
L(t)=\int \Psi(t) C(t-\tau) d \tau,
$$

where $\Psi(t)$ is the "transfer function" (TF) that describes the observed response of the emission line and depends on the geometry and reprocessing of the BLR (Blandford \& McKee 1982). The art of reverberation mapping consists of determining $\Psi(t)$ from the observables $C(t)$ and $L(t)$ and then inferring the BLR geometry. This inverse problem is notoriously difficult (see the recent book edited by Gondhalekar, Horne, \& Peterson 1994 for details) and most often the data are not numerous enough or of sufficiently high quality to do a full inversion. One therefore often restricts oneself to a simple cross-correlation analysis of the continuum and responding emission-line light curves in order to find the first moment of the TF, which coincides with the first moment of the cross-correlation function (CCF; Penston 1991; Koratkar \& Gaskell 1991). This first moment is often referred to as the "lag" and is a measure of the size of the BLR.

In this paper we analyze data obtained over six years of monitoring the Seyfert 1 galaxy Mrk 335, a type SO/a galaxy with a visual magnitude of $m_{V}=13.85$ (although it varies) and with a redshift $z=0.025$. Shuder (1981) previously showed Mrk 335 is variable in both its continuum and emission-line flux. In $\S 2$ we describe the observations and data reductions that lead to the final set of spectra, and we present and discuss the continuum and $\mathrm{H} \beta$ and $\mathrm{He}$ II emission-line light curves in $\S 3$. We perform a cross- 
correlation analysis in $\S 4$ and derive an estimate of the size of the BLR. In $\S 5$ we then examine the profile variability by means of average, root mean square, and difference spectra. Finally, $\S 6$ contains a summary of the results and the conclusions.

\section{OBSERVATIONS AND DATA REDUCTION}

The observations for this campaign were made with the Ohio State University CCD spectrograph on the Perkins $1.8 \mathrm{~m}$ Reflector of the Ohio Wesleyan and Ohio State universities at Lowell Observatory in Flagstaff, Arizona. A spectrograph entrance aperture of $5^{\prime \prime} .0 \times 7^{\prime \prime} .6$ was used and the position angle of the entrance slit was $90^{\circ}$. A grating of 350 lines $\mathrm{mm}^{-1}$ was used, giving a dispersion of $\sim 2 \AA$ per pixel and a resolution of $\sim 10 \AA$. The CCD detector used up through the 1991 observations was a Texas Instruments model 4849 chip with $384 \times 59,222 \mu$ m pixels. The detector chip used for all the observations after 1991 was a Tektronix $512 \times 512$ with $27 \mu \mathrm{m}$ pixels. A log of the spectroscopic observations for this campaign appears in Table 1. The UT date for each observation is given in column (1) and the corresponding Julian date in column (2). Column (3) lists an estimate of the seeing during the observations based on the cross-dispersion width of the broad component of $\mathrm{H} \beta$ (see Peterson et al. 1995). Column (4) lists the wavelength range covered by each observation. We will refer to the spectra by the four least significant digits in the Julian date (e.g., 7788).

The CCD frames were reduced in the standard way using the IRAF data-reduction package. This involved bias subtractions, flat-field corrections, wavelength calibrations, and standard star flux calibrations. The wavelength calibration was based on either $\mathrm{Fe}-\mathrm{Ne}$ or $\mathrm{He}-\mathrm{Ar}$ discharge tube spectra. Two or more separate 20 minute integrations of Mrk 335 were made each night it was observed so that individual exposures could be overlaid and flaws like cosmic-ray hits be identified and removed.

\subsection{Absolute Flux Calibration}

Flux calibration of ground based spectrophotometric observations of faint sources is a difficult task on account of both atmospheric transparency and seeing variations. When carried out in the standard manner, through direct use of standard stars only, the typical accuracy that can be achieved for Seyfert galaxies under good observing conditions is only about $10 \%$. Most of the continuum and emission-line variability detected on the timescales of interest (less than 1 month) is of this order or smaller, so clearly alternative calibration methods must be employed. The method that is commonly used is an "internal calibration" based upon the assumption that the narrow [O III] $\lambda \lambda 4959$, 5007 emission lines are constant in flux over the time span of the monitoring program. This is a standard method in the calibration of AGN spectra. The narrow-line region (NLR) is much larger than the BLR, hence the light travel time through the NLR is longer ( $\sim 100-1000$ years). Also, the NLR density is low enough such that recombination times are typically on the order of 100 years. These long light travel and recombination times mean the response times of the narrow lines to the continuum changes will be orders of magnitude larger than the duration of the monitoring program. A potential hazard of this method is that this assumption will fail if the NLR is spatially resolved and some of the narrow-line flux is lost at the spectrograph entrance aperture (e.g., Peterson et al. 1995). In order for the
TABLE 1

Log of SPeCtroscopic ObSERvations

\begin{tabular}{|c|c|c|c|c|}
\hline $\begin{array}{l}\text { UT Date } \\
\text { (1) }\end{array}$ & $\begin{array}{c}\text { Julian Date } \\
(2440000+) \\
(2)\end{array}$ & $\begin{array}{c}\text { Seeing } \\
\text { (arcsec) } \\
(3)\end{array}$ & $\begin{array}{c}\text { Range } \\
(\AA) \\
(4)\end{array}$ & $\begin{array}{c}\text { Integration } \\
\text { Time } \\
\text { (s) } \\
(5)\end{array}$ \\
\hline 1989 Sep 18 . & 7788.8 & 5.0 & $4580-5720$ & 2400 \\
\hline 1989 Oct $31 \ldots \ldots$ & 7831.8 & 4.8 & $4550-5632$ & 3600 \\
\hline 1989 Nov $6 . . \ldots \ldots$ & 7837.8 & 3.9 & $4590-5700$ & 3600 \\
\hline 1989 Nov $21 \ldots \ldots$ & 7852.7 & 5.5 & $4570-5720$ & 3600 \\
\hline 1989 Dec $7 \ldots \ldots$ & 7868.6 & 4.5 & $4590-5730$ & 3600 \\
\hline 1989 Dec $23 \ldots \ldots$ & 7884.6 & 3.9 & $4500-5640$ & 3600 \\
\hline 1989 Dec $30 \ldots \ldots$ & 7891.6 & 4.5 & $4500-5640$ & 2400 \\
\hline 1990 Jan $8 \ldots \ldots \ldots$ & 7900.6 & 5.6 & $4560-5700$ & 3600 \\
\hline 1990 Jan $9 . \ldots \ldots \ldots$ & 7901.6 & 2.7 & $4560-5700$ & 3600 \\
\hline 1990 Jan $23 \ldots \ldots \ldots$ & 7915.6 & 3.6 & $4560-5670$ & 2400 \\
\hline 1990 Jul $4 \ldots \ldots \ldots$ & 8078.0 & 4.0 & $4430-5570$ & 2400 \\
\hline 1990 Jul 17 . & 8091.0 & 3.1 & $4470-5610$ & 2400 \\
\hline 1990 Oct $14 \ldots \ldots$ & 8179.8 & 2.2 & $4502-5648$ & 3600 \\
\hline 1990 Oct $22 \ldots \ldots$ & 8187.8 & 2.3 & $4502-5646$ & 3600 \\
\hline 1990 Oct $28 \ldots \ldots$ & 8193.8 & 3.5 & $4514-5656$ & 3600 \\
\hline 1990 Nov 4 ....... & 8200.8 & 3.5 & $4530-5670$ & 3600 \\
\hline 1990 Nov $11 \ldots \ldots$ & 8207.7 & 2.0 & $4530-5670$ & 3600 \\
\hline 1990 Nov $24 \ldots \ldots$ & 8220.7 & 2.5 & $4540-5670$ & 3600 \\
\hline 1990 Dec $4 \ldots \ldots$ & 8230.7 & 2.5 & $4550-5670$ & 2400 \\
\hline 1990 Dec $10 \ldots \ldots$. & 8236.7 & 2.3 & $4530-5670$ & 3600 \\
\hline 1990 Dec $26 \ldots \ldots$ & 8252.7 & 5.3 & $4600-5670$ & 3600 \\
\hline 1991 Jan $18 \ldots \ldots \ldots$ & 8275.7 & 3.0 & $4600-5650$ & 2400 \\
\hline 1991 Jan $23 \ldots \ldots \ldots$ & 8280.7 & 3.0 & $4520-5660$ & 3600 \\
\hline 1991 Jan $30 \ldots \ldots$. & 8287.7 & 3.7 & $4530-5670$ & 2400 \\
\hline $1991 \mathrm{Feb} 6 \ldots \ldots \ldots$ & 8294.7 & 4.5 & $4520-5660$ & 3600 \\
\hline 1991 Sep $12 \ldots \ldots$ & 8512.8 & 4.2 & $4560-5660$ & 3600 \\
\hline 1991 Sep 13 ..... & 8513.8 & 2.5 & $4550-5650$ & 2400 \\
\hline 1991 Sep $14 \ldots \ldots$ & 8514.9 & 3.4 & $4560-5660$ & 2400 \\
\hline 1991 Sep $16 \ldots \ldots$ & 8516.8 & 3.2 & $4560-5670$ & 2400 \\
\hline 1991 Sep $20 \ldots \ldots$ & 8520.8 & 2.8 & $4540-5640$ & 3600 \\
\hline 1991 Oct $4 \ldots \ldots \ldots$ & 8534.7 & 3.2 & $4540-5640$ & 2400 \\
\hline 1992 Jul $21 \ldots \ldots$ & 8825.9 & 2.6 & $4540-5710$ & 2400 \\
\hline 1992 Jul 27 ...... & 8831.9 & 2.9 & $4580-5750$ & 3600 \\
\hline 1992 Aug 4 ...... & 8839.9 & 4.2 & $4570-5740$ & 3600 \\
\hline 1992 Aug $13 \ldots \ldots$ & 8848.9 & 1.3 & $4510-5680$ & 3600 \\
\hline 1992 Aug $27 \ldots \ldots$ & 8862.8 & 2.6 & $4560-5720$ & 4800 \\
\hline 1992 Sep $3 \ldots \ldots \ldots$ & 8869.8 & 2.7 & $4530-5690$ & 4800 \\
\hline 1992 Sep $10 \ldots \ldots$ & 8876.8 & 2.9 & $4580-5730$ & 4800 \\
\hline 1992 Sep $17 \ldots \ldots$ & 8883.8 & 3.0 & $4540-5710$ & 4800 \\
\hline 1992 Sep $23 \ldots \ldots$ & 8889.7 & 3.0 & $4500-5670$ & 3600 \\
\hline 1992 Oct $2 \ldots \ldots \ldots$ & 8898.7 & 3.2 & $4510-5670$ & 3600 \\
\hline 1992 Oct $8 \ldots \ldots \ldots$ & 8904.7 & 3.6 & $4500-5660$ & 4800 \\
\hline 1992 Oct $15 \ldots \ldots$ & 8911.7 & 3.3 & $4520-5680$ & 6000 \\
\hline 1992 Oct $22 \ldots \ldots$ & 8918.7 & 2.4 & $4590-5750$ & 6000 \\
\hline 1992 Nov $18 \ldots \ldots$ & 8945.8 & 2.6 & $4550-5700$ & 3600 \\
\hline 1992 Nov $26 . \ldots \ldots$ & 8954.7 & 2.5 & $4550-5710$ & 7200 \\
\hline 1992 Dec $9 \ldots \ldots \ldots$ & 8966.7 & 2.5 & $4520-5680$ & 3600 \\
\hline $1992 \operatorname{Dec} 23 \ldots \ldots$ & 8980.6 & 3.7 & $4500-5660$ & 3600 \\
\hline 1993 Jan $11 \ldots \ldots$ & 8999.6 & 3.5 & $4650-5670$ & 4200 \\
\hline 1993 Jun $17 \ldots \ldots$ & 9156.9 & 3.4 & $4530-5690$ & 2400 \\
\hline 1993 Jun $24 \ldots \ldots$ & 9163.9 & 2.4 & $4530-5680$ & 3600 \\
\hline 1993 Jun $30 \ldots \ldots$ & 9169.9 & 2.7 & $4530-5680$ & 4200 \\
\hline 1993 Jul $7 \ldots \ldots \ldots$ & 9176.9 & 3.0 & $4530-5690$ & 4200 \\
\hline 1993 Jul $14 \ldots \ldots \ldots$ & 9183.9 & 3.2 & $4540-5700$ & 6000 \\
\hline 1993 Jul 21 ....... & 9190.9 & 2.6 & $4560-5720$ & 2400 \\
\hline 1993 Jul 28 ....... & 9197.9 & 2.8 & $4530-5690$ & 2400 \\
\hline 1993 Aug 5 ....... & 9205.9 & 2.6 & $4540-5700$ & 4800 \\
\hline 1993 Aug $11 \ldots \ldots$ & 9211.9 & 3.3 & $4520-5680$ & 4800 \\
\hline $1993 \mathrm{Sep} 2 \ldots \ldots \ldots$ & 9233.8 & 2.3 & $4520-5690$ & 3600 \\
\hline $1993 \operatorname{Sep} 9 \ldots \ldots \ldots$ & 9240.8 & 2.5 & $4530-5690$ & 2400 \\
\hline 1993 Sep $14 \ldots \ldots$ & 9245.9 & 2.7 & $4530-5690$ & 3600 \\
\hline 1993 Sep $15 \ldots \ldots$ & 9246.8 & 2.7 & $4530-5690$ & 4200 \\
\hline 1993 Sep $22 \ldots \ldots$ & 9253.8 & 2.8 & $4520-5670$ & 3600 \\
\hline 1993 Sep $29 \ldots \ldots$ & 9260.8 & 2.7 & $4520-5670$ & 3600 \\
\hline 1993 Oct $7 \ldots \ldots \ldots$ & 9268.8 & 2.5 & $4520-5670$ & 3600 \\
\hline 1993 Oct $14 \ldots \ldots$ & 9275.7 & 2.5 & $4540-5690$ & 3600 \\
\hline 1993 Oct $21 \ldots \ldots$ & 9282.7 & 2.0 & $4520-5670$ & 4800 \\
\hline 1993 Nov 4 ....... & 9296.6 & 2.4 & $4530-5690$ & 3600 \\
\hline 1993 Nov $17 \ldots \ldots$ & 9309.7 & 2.6 & $4540-5700$ & 3600 \\
\hline 1993 Nov $24 \ldots \ldots$ & 9316.6 & 3.8 & $4520-5680$ & 3600 \\
\hline
\end{tabular}


TABLE 1-Continued

\begin{tabular}{|c|c|c|c|c|}
\hline $\begin{array}{l}\text { UT Date } \\
\text { (1) }\end{array}$ & $\begin{array}{c}\text { Julian Date } \\
(2440000+) \\
\text { (2) }\end{array}$ & $\begin{array}{c}\text { Seeing } \\
\text { (arcsec) } \\
\text { (3) }\end{array}$ & $\begin{array}{l}\text { Range } \\
(\AA ̊) \\
(4)\end{array}$ & $\begin{array}{c}\text { Integration } \\
\text { Time } \\
\text { (s) } \\
(5)\end{array}$ \\
\hline 1993 Dec 1 & 9323.6 & 2.5 & $4540-5700$ & 3600 \\
\hline 1993 Dec $10 \ldots \ldots$ & 9332.7 & 3.2 & $4520-5680$ & 3600 \\
\hline 1993 Dec $16 \ldots \ldots$ & 9338.6 & 2.6 & $4500-5660$ & 3600 \\
\hline 1994 Jun $2 \ldots \ldots \ldots$ & 9506.9 & 4.9 & $4510-5670$ & 3600 \\
\hline 1994 Jun $9 \ldots \ldots \ldots$ & 9513.9 & 3.9 & $4500-5660$ & 3600 \\
\hline 1994 Jun 16 ...... & 9520.9 & 3.7 & $4470-5630$ & 2400 \\
\hline 1994 Jun 23 ...... & 9527.9 & 3.5 & $4540-5700$ & 3600 \\
\hline 1994 Jun 30 ...... & 9534.9 & 5.4 & $4510-5670$ & 1200 \\
\hline 1994 Sep 1 ........ & 9597.8 & 2.2 & $4510-5670$ & 3600 \\
\hline 1994 Sep $12 \ldots \ldots$ & 9608.8 & 2.1 & $4510-5680$ & 3600 \\
\hline 1994 Sep $26 \ldots \ldots$ & 9622.8 & 3.0 & $4520-5690$ & 3600 \\
\hline 1994 Oct $11 \ldots \ldots$ & 9637.7 & 3.1 & $4530-5690$ & 3600 \\
\hline 1994 Oct $16 \ldots \ldots$ & 9642.8 & 3.4 & $4500-5680$ & 3600 \\
\hline 1994 Oct $23 \ldots \ldots$ & 9649.8 & 1.8 & $4510-5680$ & 3600 \\
\hline 1994 Nov 1 ....... & 9658.6 & 2.4 & $4510-5680$ & 3600 \\
\hline 1994 Nov 7 ....... & 9664.7 & 3.0 & $4510-5680$ & 3600 \\
\hline 1994 Nov $14 \ldots \ldots$ & 9671.7 & 3.5 & $4590-5750$ & 3600 \\
\hline 1994 Nov $21 \ldots \ldots$ & 9678.7 & 2.2 & $4510-5680$ & 3600 \\
\hline 1994 Nov $28 \ldots \ldots$ & 9685.7 & 3.2 & $4510-5680$ & 3600 \\
\hline 1995 Feb $2 \ldots \ldots \ldots$ & 9751.6 & 2.8 & $4500-5660$ & 3600 \\
\hline
\end{tabular}

narrow lines to be effective internal flux calibrators, we need to ensure that under all seeing conditions the fraction of light lost at the entrance aperture is nearly the same for the narrow lines and for the broad lines and AGN continuum, which arise in pointlike sources. This is achieved through use of an effective aperture somewhat larger than the poorest seeing we expect to encounter. The effective entrance aperture we use is $55^{\prime \prime} 0 \times 77^{\prime \prime} 6$.

We use the automated scaling program developed by van Groningen \& Wanders (1992) to flux-calibrate the spectra in an objective way. The scaling algorithm introduces a flux scaling factor (a multiplicative "gray" scaling), a zero-point wavelength shift, and a small amount of smoothing (with a Gaussian filter) to yield minimum residuals at the position of the [O III] lines in the difference spectra formed by subtracting a "reference" spectrum from each observed spectrum. In this way, small differences in wavelength calibration, flux calibration, and spectral resolution between the observed spectra (after the standard reduction procedures) are corrected for. Each spectrum is thus scaled to a particular reference spectrum. The earlier (1989-1991) data obtained with the TI CCD are of somewhat lower quality than the more recent data obtained with the Tektronix CCD, so a reference spectrum for the earlier set was formed by combining all the data to make an average spectrum. The spectrum chosen to be the reference spectrum for the more recent data (1992-1994) was taken on 1993 September 15. The algorithm takes the spectrum to be scaled and subtracts the reference spectrum to obtain a "difference spectrum." A second-order polynomial is then fitted to the difference spectrum in a wavelength range around the [O III] lines, and a $\chi^{2}$ goodness of fit is computed. The scaling parameters for which $\chi^{2}$ is minimized are taken as "best," and the spectrum is accordingly scaled. The wavelength range over which the $\chi^{2}$ minimization is performed preferably includes both [O III] lines, and has as little $\mathrm{H} \beta$ contamination as possible. The wavelength range used for this data set is 5066-5230 $\AA$ (in the observer's rest frame). When the spectrum is correctly scaled, there should be no trace of the [O III] lines in the difference spectrum. This scaling procedure produces a homogeneous data set. As a final check on the integrity of the scaling process, we compared the observed continuum and line flux variations with the scaling factors and find no evidence for any correlation.

\section{THE LIGHT CURVES}

Once the spectra are calibrated with respect to each other, the flux from the continuum and the $\mathrm{H} \beta$ and $\mathrm{He}$ II $\lambda 4686$ emission lines can be measured from each observation. The continuum flux was simply measured as the average flux over the observed wavelength region 5220 $5240 \AA$ A. The total $\mathrm{H} \beta$ line flux was measured by identifying the wavelength range containing the spectral line and interpolating a linear pseudo-continuum underneath the line. The pseudo-continuum is a straight line, defined by the continuum on either side of the line. The $\mathrm{H} \beta$ flux is then the sum of the flux in that wavelength range above the pseudocontinuum. The wavelength range over which the $\mathrm{H} \beta$ flux was integrated for this set of spectra was $4900-5040 \AA$, in the observer's rest frame. The He II line flux was measured in the same manner as the $\mathrm{H} \beta$ line flux, though over the wavelength range $4753-4850 \AA$ in the observer's rest frame.

All of the measurements made from the scaled spectra are on a relative scale: relative to the reference spectrum to which the spectrum is scaled. To place the flux measurements on an absolute scale, we use standard spectroscopic techniques to determine the absolute flux in the [O III] $\lambda 5007$ line and then determine a multiplicative scale factor that will yield this flux for [O III] $\lambda 5007$ in the reference spectrum. All flux measurements are then multiplied by this factor to obtain an absolute scale. The absolute flux in [O III] $\lambda 5007$ was determined by averaging the measurements made from all spectra that were obtained under photometric conditions. We judged spectra to be of photometric quality if (1) the observer judged that the atmospheric transparency was excellent and stable; (2) the count levels in the calibration spectra on different standard stars agreed to within $\sim 5 \%$ over the course of the night; and (3) the count levels in the individual spectra of Mrk 335 that were taken on the same night agreed to within a few percent. The mean value for the [O III] $\lambda 5007$ flux from these photometric nights is $(2.40 \pm 0.14) \times 10^{-13} \mathrm{erg} \mathrm{cm}^{-2}$ $\mathrm{s}^{-1}$, and this value is adopted as the absolute flux of the [O III] $\lambda 5007$ line, in the observer's frame. The values for the continuum, $\mathrm{H} \beta$, and $\mathrm{He}$ II absolute fluxes based on this absolute calibration are listed in Table 2, along with the flux scaling factors introduced by the scaling algorithm. The uncertainties in these measurements were estimated by comparing pairs of observations made within a few $(<5)$ days of each other (e.g., Peterson et al. 1991, 1993). The estimated errors are $2 \%$ of each continuum and $\mathrm{H} \beta$ measurement and $7 \%$ of each $\mathrm{He}$ II measurement. We have thus produced the continuum, $\mathrm{H} \beta$, and $\mathrm{He}$ II light curves, given in Table 2 and shown in Figure 1.

The continuum, $\mathrm{H} \beta$, and $\mathrm{He}$ II light curves clearly show that the continuum and the $\mathrm{H} \beta$ and $\mathrm{He}$ II emission lines vary according to a similar pattern. Table 3 gives a summary of some of the characteristics of the variability and sampling of the data base for each year of monitoring. These characteristics include the parameters $F_{\text {var }}$ and $R_{\max }$, which are the root mean square (rms) fluctuation amplitude relative to the mean flux and the ratio of maximum to 
TABLE 2

Continuum, $\mathrm{H} \beta$, AND He il Light CuRves

\begin{tabular}{|c|c|c|c|c|}
\hline $\begin{array}{l}\text { Julian Date } \\
(2440000+) \\
\text { (1) }\end{array}$ & $\begin{array}{l}\text { Scaling Factor } \\
\text { (2) }\end{array}$ & $\begin{array}{c}F_{\lambda}(5100 \AA) \\
\left(10^{-15} \mathrm{erg} \mathrm{s}^{-1} \mathrm{~cm}^{-2} \AA^{-1}\right) \\
(3)\end{array}$ & $\begin{array}{c}F(\mathrm{H} \beta) \\
\left(10^{-13} \mathrm{erg} \mathrm{s}^{-1} \mathrm{~cm}^{-2}\right)\end{array}$ & $\begin{array}{c}F(\mathrm{He} \mathrm{II} \lambda 4686) \\
\left(10^{-13} \mathrm{erg} \mathrm{s}^{-1} \mathrm{~cm}^{-2}\right) \\
(5)\end{array}$ \\
\hline $7788.8 \ldots \ldots$ & 1.071 & $9.63 \pm 0.19$ & $8.80 \pm 0.18$ & $1.85 \pm 0.04$ \\
\hline $7831.8 \ldots \ldots$ & 1.007 & $8.74 \pm 0.18$ & $8.00 \pm 0.16$ & $1.54 \pm 0.03$ \\
\hline $7837.8 \ldots \ldots$ & 1.003 & $8.13 \pm 0.16$ & $7.88 \pm 0.16$ & $1.52 \pm 0.03$ \\
\hline $7852.7 \ldots \ldots$ & 1.037 & $8.70 \pm 0.17$ & $7.72 \pm 0.15$ & $1.88 \pm 0.04$ \\
\hline $7868.6 \ldots \ldots$ & 1.120 & $9.05 \pm 0.18$ & $8.23 \pm 0.17$ & $1.97 \pm 0.04$ \\
\hline $7884.6 \ldots \ldots$ & 1.056 & $8.97 \pm 0.18$ & $8.09 \pm 0.16$ & $2.01 \pm 0.04$ \\
\hline $7891.6 \ldots \ldots$ & 0.889 & $9.46 \pm 0.19$ & $8.62 \pm 0.17$ & $2.25 \pm 0.05$ \\
\hline $7900.6 \ldots \ldots$ & 1.212 & $8.88 \pm 0.18$ & $8.80 \pm 0.18$ & $1.82 \pm 0.04$ \\
\hline $7901.6 \ldots \ldots$ & 1.085 & $8.79 \pm 0.18$ & $8.59 \pm 0.17$ & $1.74 \pm 0.04$ \\
\hline $7915.6 \ldots \ldots$ & 1.022 & $8.71 \pm 0.17$ & $8.33 \pm 0.17$ & $1.87 \pm 0.04$ \\
\hline $8078.0 \ldots \ldots$ & 0.803 & $8.10 \pm 0.16$ & $7.65 \pm 0.15$ & $1.84 \pm 0.04$ \\
\hline $8091.0 \ldots \ldots$ & 0.968 & $7.92 \pm 0.16$ & $7.57 \pm 0.15$ & $1.52 \pm 0.03$ \\
\hline $8179.8 \ldots \ldots$ & 0.990 & $9.37 \pm 0.19$ & $7.65 \pm 0.15$ & $2.07 \pm 0.04$ \\
\hline $8187.8 \ldots \ldots$ & 0.969 & $9.63 \pm 0.19$ & $8.32 \pm 0.17$ & $1.89 \pm 0.04$ \\
\hline $8193.8 \ldots \ldots$ & 1.019 & $9.77 \pm 0.20$ & $8.40 \pm 0.17$ & $2.17 \pm 0.04$ \\
\hline $8200.8 \ldots \ldots$ & 0.982 & $9.37 \pm 0.19$ & $8.39 \pm 0.17$ & $1.74 \pm 0.04$ \\
\hline $8207.7 \ldots \ldots$ & 0.970 & $9.39 \pm 0.19$ & $8.40 \pm 0.17$ & $1.82 \pm 0.04$ \\
\hline $8220.7 \ldots \ldots$ & 0.974 & $8.79 \pm 0.18$ & $8.24 \pm 0.17$ & $1.56 \pm 0.03$ \\
\hline $8230.7 \ldots \ldots$ & 0.998 & $8.83 \pm 0.18$ & $8.31 \pm 0.17$ & $1.54 \pm 0.03$ \\
\hline $8236.7 \ldots \ldots$ & 0.981 & $9.09 \pm 0.18$ & $8.10 \pm 0.16$ & $1.80 \pm 0.04$ \\
\hline $8252.7 \ldots \ldots$ & 0.976 & $8.65 \pm 0.17$ & $7.74 \pm 0.16$ & $1.55 \pm 0.03$ \\
\hline $8275.7 \ldots \ldots$ & 0.914 & $7.77 \pm 0.16$ & $7.38 \pm 0.15$ & $1.23 \pm 0.03$ \\
\hline $8280.7 \ldots \ldots$ & 0.999 & $8.29 \pm 0.17$ & $7.23 \pm 0.15$ & $1.42 \pm 0.03$ \\
\hline $8287.7 \ldots \ldots$ & 1.164 & $8.78 \pm 0.18$ & $7.52 \pm 0.15$ & $1.57 \pm 0.03$ \\
\hline $8294.7 \ldots \ldots$ & 0.934 & $8.89 \pm 0.18$ & $7.64 \pm 0.15$ & $1.81 \pm 0.04$ \\
\hline $8512.8 \ldots \ldots$ & 0.904 & $8.60 \pm 0.17$ & $7.19 \pm 0.14$ & $1.43 \pm 0.03$ \\
\hline $8513.8 \ldots \ldots$ & 0.889 & $8.36 \pm 0.17$ & $6.96 \pm 0.14$ & $1.15 \pm 0.02$ \\
\hline $8514.9 \ldots \ldots$ & 0.882 & $8.22 \pm 0.16$ & $6.81 \pm 0.14$ & $0.93 \pm 0.02$ \\
\hline $8516.8 \ldots \ldots$ & 0.969 & $8.25 \pm 0.17$ & $7.17 \pm 0.14$ & $1.19 \pm 0.02$ \\
\hline $8520.8 \ldots \ldots$ & 0.937 & $8.46 \pm 0.17$ & $7.28 \pm 0.15$ & $1.12 \pm 0.02$ \\
\hline $8534.7 \ldots \ldots$ & 0.965 & $8.36 \pm 0.17$ & $7.10 \pm 0.14$ & $1.29 \pm 0.03$ \\
\hline $8825.9 \ldots \ldots$ & 0.982 & $9.66 \pm 0.19$ & $9.41 \pm 0.19$ & $2.64 \pm 0.05$ \\
\hline $8831.9 \ldots \ldots$ & 0.997 & $9.21 \pm 0.18$ & $9.47 \pm 0.19$ & $2.41 \pm 0.05$ \\
\hline $8839.9 \ldots \ldots$ & 1.261 & $8.96 \pm 0.18$ & $9.19 \pm 0.18$ & $2.45 \pm 0.05$ \\
\hline $8848.9 \ldots \ldots$ & 0.972 & $9.15 \pm 0.18$ & $8.90 \pm 0.18$ & $1.97 \pm 0.04$ \\
\hline $8862.8 \ldots \ldots$ & 0.962 & $8.60 \pm 0.17$ & $8.87 \pm 0.18$ & $1.97 \pm 0.04$ \\
\hline $8869.8 \ldots \ldots$ & 0.943 & $8.94 \pm 0.18$ & $8.58 \pm 0.17$ & $2.16 \pm 0.04$ \\
\hline $8876.8 \ldots \ldots$ & 1.053 & $9.21 \pm 0.18$ & $8.80 \pm 0.18$ & $2.25 \pm 0.05$ \\
\hline $8883.8 \ldots \ldots$ & 0.991 & $9.06 \pm 0.18$ & $8.82 \pm 0.18$ & $2.16 \pm 0.04$ \\
\hline $8889.7 \ldots \ldots$ & 0.961 & $8.94 \pm 0.18$ & $8.94 \pm 0.18$ & $2.14 \pm 0.04$ \\
\hline $8898.7 \ldots \ldots$ & 0.975 & $9.27 \pm 0.19$ & $9.03 \pm 0.18$ & $2.37 \pm 0.05$ \\
\hline $8904.7 \ldots \ldots$ & 0.919 & $8.94 \pm 0.18$ & $8.94 \pm 0.18$ & $2.00 \pm 0.04$ \\
\hline $8911.7 \ldots \ldots$ & 0.963 & $9.31 \pm 0.19$ & $9.18 \pm 0.18$ & $2.35 \pm 0.05$ \\
\hline $8918.7 \ldots \ldots$ & 0.878 & $9.04 \pm 0.18$ & $8.84 \pm 0.18$ & $2.06 \pm 0.04$ \\
\hline $8945.8 \ldots \ldots$ & 0.920 & $7.70 \pm 0.15$ & $8.31 \pm 0.17$ & $1.46 \pm 0.03$ \\
\hline $8954.7 \ldots \ldots$ & 0.954 & $8.15 \pm 0.16$ & $8.40 \pm 0.17$ & $1.61 \pm 0.03$ \\
\hline $8966.7 \ldots \ldots$ & 0.914 & $8.11 \pm 0.16$ & $8.37 \pm 0.17$ & $1.67 \pm 0.03$ \\
\hline $8980.6 \ldots \ldots$ & 0.969 & $8.57 \pm 0.17$ & $8.47 \pm 0.17$ & $2.05 \pm 0.04$ \\
\hline $8999.6 \ldots \ldots$ & 0.931 & $8.40 \pm 0.17$ & $8.54 \pm 0.17$ & $1.82 \pm 0.04$ \\
\hline $9156.9 \ldots \ldots$ & 1.005 & $8.25 \pm 0.17$ & $8.03 \pm 0.16$ & $1.64 \pm 0.03$ \\
\hline $9163.9 \ldots \ldots$ & 0.971 & $8.16 \pm 0.16$ & $8.07 \pm 0.16$ & $1.94 \pm 0.04$ \\
\hline $9169.9 \ldots \ldots$ & 0.981 & $8.02 \pm 0.16$ & $8.30 \pm 0.17$ & $1.90 \pm 0.04$ \\
\hline $9176.9 \ldots \ldots$ & 0.977 & $7.89 \pm 0.16$ & $8.24 \pm 0.17$ & $1.77 \pm 0.04$ \\
\hline $9183.9 \ldots \ldots$ & 1.055 & $7.75 \pm 0.16$ & $8.12 \pm 0.16$ & $1.81 \pm 0.04$ \\
\hline $9190.9 \ldots \ldots$ & 0.790 & $7.15 \pm 0.14$ & $7.70 \pm 0.15$ & $1.50 \pm 0.03$ \\
\hline $9197.9 \ldots \ldots$ & 0.753 & $7.23 \pm 0.15$ & $7.75 \pm 0.16$ & $1.67 \pm 0.03$ \\
\hline $9205.9 \ldots \ldots$ & 0.950 & $7.21 \pm 0.14$ & $7.84 \pm 0.16$ & $1.54 \pm 0.03$ \\
\hline $9211.9 \ldots \ldots$ & 1.472 & $6.98 \pm 0.14$ & $7.30 \pm 0.15$ & $1.51 \pm 0.03$ \\
\hline $9233.8 \ldots \ldots$ & 0.939 & $7.27 \pm 0.15$ & $7.37 \pm 0.15$ & $1.40 \pm 0.03$ \\
\hline $9240.8 \ldots \ldots$ & 0.956 & $7.72 \pm 0.15$ & $7.44 \pm 0.15$ & $1.50 \pm 0.03$ \\
\hline $9245.9 \ldots \ldots$ & 0.962 & $7.89 \pm 0.16$ & $7.45 \pm 0.15$ & $1.75 \pm 0.04$ \\
\hline $9246.8 \ldots \ldots$ & 1.000 & $8.14 \pm 0.16$ & $7.70 \pm 0.15$ & $1.81 \pm 0.04$ \\
\hline $9253.8 \ldots \ldots$ & 1.000 & $8.18 \pm 0.16$ & $7.76 \pm 0.16$ & $2.13 \pm 0.04$ \\
\hline $9260.8 \ldots \ldots$ & 0.961 & $8.14 \pm 0.16$ & $7.75 \pm 0.16$ & $2.30 \pm 0.05$ \\
\hline $9268.8 \ldots \ldots$ & 0.948 & $8.68 \pm 0.17$ & $8.06 \pm 0.16$ & $2.35 \pm 0.05$ \\
\hline $9275.7 \ldots \ldots$ & 0.965 & $8.92 \pm 0.18$ & $8.16 \pm 0.16$ & $2.42 \pm 0.05$ \\
\hline $9282.7 \ldots \ldots$ & 0.812 & $8.60 \pm 0.17$ & $8.71 \pm 0.17$ & $2.31 \pm 0.05$ \\
\hline $9296.6 \ldots \ldots$ & 0.904 & $7.98 \pm 0.16$ & $8.31 \pm 0.17$ & $1.75 \pm 0.04$ \\
\hline $9309.7 \ldots \ldots$ & 0.945 & $7.66 \pm 0.15$ & $8.04 \pm 0.16$ & $1.61 \pm 0.03$ \\
\hline $9316.6 \ldots \ldots$ & 0.909 & $7.66 \pm 0.15$ & $8.21 \pm 0.16$ & $1.46 \pm 0.03$ \\
\hline
\end{tabular}


TABLE 2-Continued

\begin{tabular}{|c|c|c|c|c|}
\hline $\begin{array}{l}\text { Julian Date } \\
(2440000+) \\
\text { (1) }\end{array}$ & $\begin{array}{l}\text { Scaling Factor } \\
\text { (2) }\end{array}$ & $\begin{array}{c}F_{\lambda}(5100 \AA) \\
\left(10^{-15} \mathrm{erg} \mathrm{s}^{-1} \mathrm{~cm}^{-2} \AA^{-1}\right) \\
(3)\end{array}$ & $\begin{array}{c}F(\mathrm{H} \beta) \\
\left(10^{-13} \mathrm{erg} \mathrm{s}^{-1} \mathrm{~cm}^{-2}\right) \\
(4)\end{array}$ & $\begin{array}{c}F(\mathrm{He} \mathrm{II} \lambda 4686) \\
\left(10^{-13} \mathrm{erg} \mathrm{s}^{-1} \mathrm{~cm}^{-2}\right) \\
(5)\end{array}$ \\
\hline $9323.6 \ldots \ldots$ & 0.938 & $7.42 \pm 0.15$ & $7.83 \pm 0.16$ & $1.51 \pm 0.03$ \\
\hline $9332.7 \ldots \ldots$ & 0.904 & $7.26 \pm 0.15$ & $7.62 \pm 0.15$ & $1.54 \pm 0.03$ \\
\hline $9338.6 \ldots \ldots$ & 0.907 & $7.31 \pm 0.15$ & $7.84 \pm 0.16$ & $1.66 \pm 0.03$ \\
\hline $9506.9 \ldots \ldots$ & 1.138 & $6.89 \pm 0.14$ & $7.33 \pm 0.15$ & $1.77 \pm 0.04$ \\
\hline $9513.9 \ldots \ldots$ & 1.109 & $7.09 \pm 0.14$ & $7.39 \pm 0.15$ & $1.66 \pm 0.03$ \\
\hline $9520.9 \ldots \ldots$ & 4.015 & $6.63 \pm 0.13$ & $7.40 \pm 0.15$ & $1.69 \pm 0.03$ \\
\hline $9527.9 \ldots \ldots$ & 1.073 & $6.93 \pm 0.14$ & $6.99 \pm 0.14$ & $1.42 \pm 0.03$ \\
\hline $9534.9 \ldots \ldots$ & 4.431 & $7.44 \pm 0.15$ & $7.58 \pm 0.15$ & $1.81 \pm 0.04$ \\
\hline $9597.8 \ldots \ldots$ & 1.051 & $8.26 \pm 0.17$ & $7.87 \pm 0.16$ & $2.31 \pm 0.05$ \\
\hline $9608.8 \ldots \ldots$ & 1.010 & $8.33 \pm 0.17$ & $7.84 \pm 0.16$ & $2.41 \pm 0.05$ \\
\hline $9622.8 \ldots \ldots$ & 0.958 & $8.14 \pm 0.16$ & $8.35 \pm 0.17$ & $2.08 \pm 0.04$ \\
\hline $9637.7 \ldots \ldots$ & 0.982 & $8.02 \pm 0.16$ & $8.32 \pm 0.17$ & $2.08 \pm 0.04$ \\
\hline $9642.8 \ldots \ldots$ & 0.985 & $8.56 \pm 0.17$ & $8.17 \pm 0.16$ & $2.25 \pm 0.05$ \\
\hline $9649.8 \ldots \ldots$ & 0.933 & $8.02 \pm 0.16$ & $8.05 \pm 0.16$ & $2.23 \pm 0.05$ \\
\hline $9658.6 \ldots \ldots$ & 0.983 & $7.96 \pm 0.16$ & $8.53 \pm 0.17$ & $1.88 \pm 0.04$ \\
\hline $9664.7 \ldots \ldots$ & 0.719 & $7.88 \pm 0.16$ & $8.17 \pm 0.16$ & $1.99 \pm 0.04$ \\
\hline $9671.7 \ldots \ldots$ & 1.038 & $7.59 \pm 0.15$ & $7.67 \pm 0.15$ & $1.74 \pm 0.04$ \\
\hline 9678.7. & 1.074 & $7.81 \pm 0.16$ & $8.22 \pm 0.16$ & $1.55 \pm 0.03$ \\
\hline $9685.7 \ldots \ldots$ & 1.018 & $7.75 \pm 0.16$ & $7.91 \pm 0.16$ & $1.62 \pm 0.03$ \\
\hline $9751.6 \ldots \ldots$ & 0.960 & $8.04 \pm 0.16$ & $8.58 \pm 0.17$ & $1.84 \pm 0.04$ \\
\hline
\end{tabular}

minimum flux, respectively (Clavel et al. 1991).

\section{ANALYSIS OF THE VARIATIONS}

The time delay between continuum and emission-line variations - the "lag" - can be determined by cross- correlating the continuum light curve and the $\mathrm{H} \beta$ and $\mathrm{He}$ II light curves. The cross-correlations of the light curves are carried out using the interpolation method described by Gaskell \& Peterson (1987) and with the discrete correlation method (DCF) of Edelson \& Krolik (1988). The specific

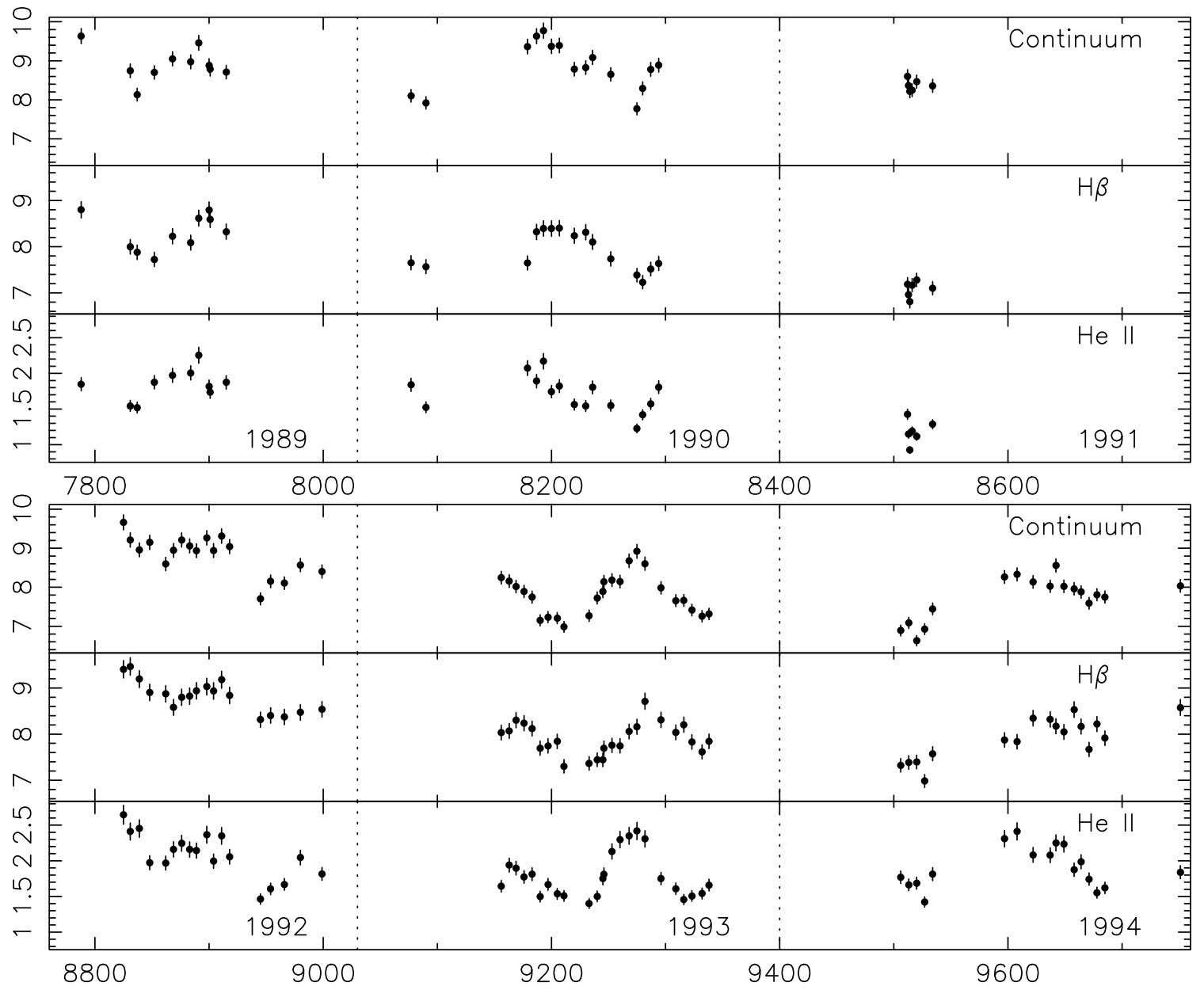

FIg. 1.-Continuum, $\mathrm{H} \beta$, and He II light curves from 1989 to 1994. The continuum fluxes are in units of $10^{-15} \mathrm{erg} \mathrm{s}^{-1} \mathrm{~cm}^{-2} \AA^{-1}$, and the line fluxes are in units of $10^{-13} \mathrm{erg} \mathrm{s}^{-1} \mathrm{~cm}^{-2}$. 
TABLE 3

SAMPling Characteristics AND VARIABiLITy PARAMETERS

\begin{tabular}{|c|c|c|c|c|c|c|c|c|}
\hline $\begin{array}{c}\text { Time Interval } \\
\text { (1) }\end{array}$ & $\begin{array}{l}\text { Feature } \\
\text { (2) }\end{array}$ & $\begin{array}{l}\text { Number of Epochs } \\
\text { (3) }\end{array}$ & $\begin{array}{l}\text { Total Span } \\
\text { (days) } \\
\text { (4) }\end{array}$ & $\begin{array}{l}\text { Average Sampling } \\
\text { (days) } \\
\text { (5) }\end{array}$ & $\begin{array}{l}\text { Median Sampling } \\
\text { (days) } \\
\text { (6) }\end{array}$ & $\begin{array}{l}\text { Mean Flux } \\
\text { (7) }\end{array}$ & $\begin{array}{l}F_{\mathrm{var}} \\
(8)\end{array}$ & $\begin{array}{l}R_{\max } \\
(9)\end{array}$ \\
\hline \multirow[t]{3}{*}{$1989 \ldots \ldots \ldots \ldots$} & $F_{\lambda}(5110 \AA)$ & 10 & 127 & 14.1 & 14.0 & 8.906 & 0.040 & 1.184 \\
\hline & $\mathrm{H} \beta$ & 10 & 127 & 14.1 & 14.0 & 8.304 & 0.039 & 1.139 \\
\hline & He II & 10 & 127 & 14.1 & 14.0 & 1.845 & 0.110 & 1.482 \\
\hline \multirow[t]{3}{*}{$1990 \ldots \ldots \ldots \ldots$} & $F_{\lambda}(5110 \AA)$ & 15 & 217 & 15.5 & 7.5 & 8.842 & 0.064 & 1.258 \\
\hline & $\mathrm{H} \beta$ & 15 & 217 & 15.5 & 7.5 & 7.903 & 0.047 & 1.161 \\
\hline & $\mathrm{He}$ II & 15 & 217 & 15.5 & 7.5 & 1.703 & 0.141 & 1.768 \\
\hline \multirow[t]{3}{*}{$1991 \ldots \ldots \ldots \ldots$} & $F_{\lambda}(5110 \AA)$ & 6 & 22 & 3.7 & 2.0 & 8.374 & 0.007 & 1.047 \\
\hline & $\mathrm{H} \beta$ & 6 & 22 & 3.7 & 2.0 & 7.084 & 0.014 & 1.069 \\
\hline & He II & 6 & 22 & 3.7 & 2.0 & 1.182 & 0.133 & 1.541 \\
\hline \multirow[t]{3}{*}{$1992 \ldots \ldots \ldots \ldots$} & $F_{\lambda}(5110 \AA)$ & 18 & 174 & 10.2 & 8.0 & 8.845 & 0.051 & 1.255 \\
\hline & $\mathrm{H} \beta$ & 18 & 174 & 10.2 & 8.0 & 8.836 & 0.034 & 1.139 \\
\hline & He II & 18 & 174 & 10.2 & 8.0 & 2.084 & 0.144 & 1.806 \\
\hline \multirow[t]{3}{*}{$1993 \ldots \ldots \ldots \ldots$} & $F_{\lambda}(5110 \AA)$ & 24 & 182 & 7.9 & 7.0 & 7.811 & 0.062 & 1.277 \\
\hline & $\mathrm{H} \beta$ & 24 & 182 & 7.9 & 7.0 & 7.899 & 0.039 & 1.193 \\
\hline & He II & 24 & 182 & 7.9 & 7.0 & 1.782 & 0.169 & 1.726 \\
\hline \multirow[t]{3}{*}{$1994 \ldots \ldots \ldots \ldots$} & $F_{\lambda}(5110 \AA)$ & 17 & 245 & 15.3 & 7.0 & 7.725 & 0.067 & 1.291 \\
\hline & $\mathbf{H} \beta$ & 17 & 245 & 15.3 & 7.0 & 7.902 & 0.053 & 1.228 \\
\hline & He II & 17 & 245 & 15.3 & 7.0 & 1.902 & 0.146 & 1.695 \\
\hline \multirow[t]{3}{*}{ Combined...... } & $F_{\lambda}(5110 \AA)$ & 90 & 1963 & 22.1 & 7.0 & 8.333 & 0.085 & 1.474 \\
\hline & $\mathrm{H} \beta$ & 90 & 1963 & 22.1 & 7.0 & 8.078 & 0.070 & 1.390 \\
\hline & $\mathrm{He}$ II & 90 & 1963 & 22.1 & 7.0 & 1.819 & 0.188 & 2.855 \\
\hline
\end{tabular}

algorithms used are those described by White \& Peterson (1994). When computing the cross-correction functions for the 1990 data set, the first two observations were ignored due to the large gap in the time between those observations and the others in that year. Undersampling, large gaps in the time range of the light curves, or the lack of distinctive variations (i.e., an "event") produced somewhat ambiguous results in the cross-correlations of many of the data subsets, except in the case of the 1993 data, where two clean inflection points are observed in both the continuum and the lines (see Fig. 1). The CCFs and DCFs of the $\mathrm{H} \beta$ and He II light curves of the 1993 data set, along with the continuum

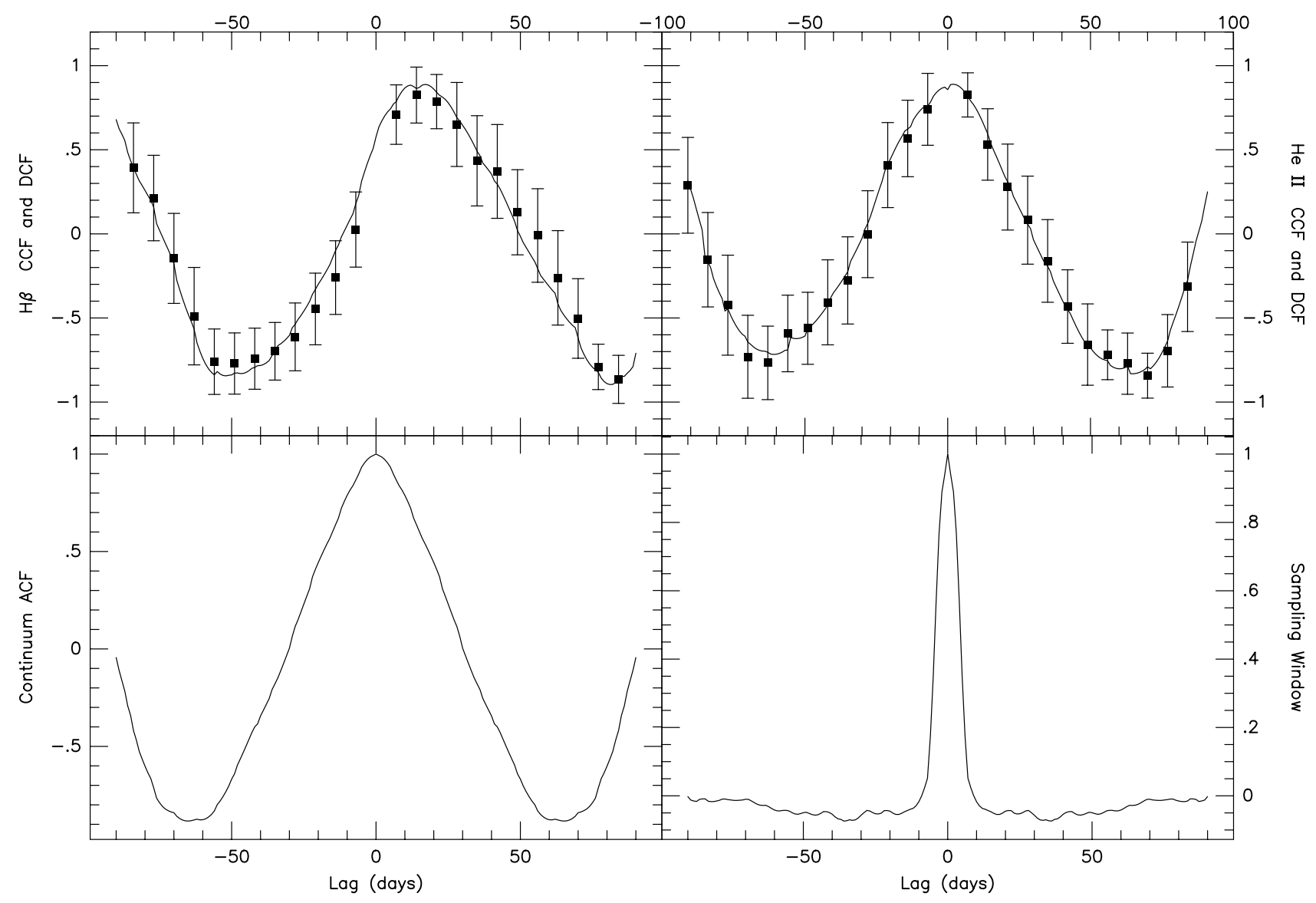

FIG. 2.- $\mathrm{H} \beta$ and $\mathrm{He}$ II cross-correlation functions, the continuum autocorrelation function, and the sampling window function 
TABLE 4

Cross-Correlation Results For 1993 Data

\begin{tabular}{ccc}
\hline \hline $\begin{array}{c}\text { Parameter } \\
(1)\end{array}$ & $\mathrm{H} \beta$ Value & He II Value \\
$(2)$ & $(3)$ \\
\hline$\Delta t_{\text {peak }}$ (days) $\ldots \ldots . .$. & 17.0 & 2.0 \\
$\Delta t_{\text {cent }}$ (days) $\ldots \ldots$. & 16.6 & -1.1 \\
$r_{\max } \ldots \ldots \ldots \ldots \ldots$ & 0.89 & 0.89 \\
\hline
\end{tabular}

autocorrelation function and the sampling window function, are shown in Figure 2. A summary of these results is given in Table 4. The peak value of the CCF for the continuum and $\mathrm{H} \beta$ light curves was found to be $17 \pm 3$ days. For the continuum and He II light curves, the peak value of the CCF was $2 \pm 3$ days. The 3 day uncertainty was assigned based on Monte Carlo simulations (Peterson et al. 1991). The value $r_{\text {max }}$, given in Table 4, is the maximum correlation coefficient at these lags. The centroid of the CCF (based on points with $r \geq 0.5 r_{\max }$ ) for the continuum and $\mathrm{H} \beta$ light curves was at $17 \pm 3$ days and for the continuum and He II light curves at $-1 \pm 3$ days. It is thus observed that the $\mathrm{He}$ II line responds virtually instantaneously to continuum variations, whereas the $\mathrm{H} \beta$ line shows a significant lag.

The lag is a measure of the size of the BLR (Blandford \& McKee 1982) and if the BLR consists of gas spherically distributed around the continuum source, the derived lags for $\mathrm{H} \beta$ and $\mathrm{He}$ II imply that the $\mathrm{H} \beta$ emitting region is on the order of 17 light-days in radius and the $\mathrm{He}$ II emitting region less than about 2 light-days. This result is consistent with radial ionization stratification, as inferred for other AGNs and demonstrates that the BLR geometry is different for each line.

\section{PROFILE VARIATIONS}

The quality of the data set is good enough to do a more extensive analysis using profile variability rather than examining the total line fluxes only, as done hitherto.

\subsection{Average and Root Mean Square Spectra}

Good measures of profile variability are the average and root mean square (rms) spectra, $\phi(v)$ and $\sigma(v)$, respectively, which are defined in the usual statistical sense:

$$
\begin{gathered}
\phi(v)=\frac{1}{N} \sum_{i=1}^{N} L\left(v, t_{i}\right), \\
\sigma(v)=\frac{1}{(N-1)} \sum_{i=1}^{N}\left[L\left(v, t_{i}\right)-\phi(v)\right]^{2} .
\end{gathered}
$$

Here $N$ is the number of emission-line profiles $L\left(v, t_{i}\right)$, each observed at time $t_{i}$.

Figure 3 presents the average and rms spectra for the full data set, as well as for each individual monitoring year. Any constant contributions to the spectra such as the narrow emission lines will not show up in the rms spectra. The rms spectrum is thus a good indicator of variable parts of the spectrum.

The average profiles do not show much structure in the $\mathrm{H} \beta$ and $\mathrm{He}$ II profiles. No attempt has been made to subtract the narrow $\mathrm{H} \beta$ and $\mathrm{He}$ II lines because of their blending with the underlying broad profiles. However, as can be judged from the relative weakness of the narrow [O III] lines, the narrow $\mathrm{H} \beta$ and $\mathrm{He}$ II lines, which are generally much weaker than the [O III] lines in Seyfert 1 galaxies, will not make a significant contribution to the total emission line.

The full width at zero intensity (FWZI) of the $\mathrm{H} \beta \mathrm{rms}$ profiles is smaller than the FWZI of the $\mathrm{H} \beta$ average profiles, indicating that the variations in the $\mathrm{H} \beta$ profile take place mainly in the core of the line and not in the far wings. Interestingly, the He II line varies over its full width. The well-sampled and eventful year 1993 shows the large differences in FWZI in the $\mathrm{H} \beta$ and He II lines in the rms spectra especially well.

There is a small slope visible in the continuum flux rms spectrum, in the sense that the variations in the blue part of the spectrum are larger than in the red part of the spectrum. This means the continuum spectrum becomes harder when it brightens, which is apparently a general characteristic of AGNs (e.g., Edelson, Krolik, \& Pike 1990).

The small spikes in the rms spectra that correspond to the wavelength of the strong [O III] $\lambda 5007$ line arise due to the fact that the calculation of the rms spectrum involves taking the difference between a single spectrum and the average spectrum. Because the average spectrum is the sum of a large number of individual spectra with tiny wavelength calibration errors, the resolution of the average spectrum will be slightly less than that of the individual spectra. Hence, a sharp feature in a spectrum will give a slight residual in the rms spectra. The sharp peak in the $1994 \mathrm{rms}$ spectrum at about $5040 \AA$ is due to an unremoved cosmicray hit in one of the spectra and is not an intrinsic feature of Markarian 335.

\subsection{Difference Spectra}

In Figures $4 a-4 f$ the differences between the individual spectra and the average of the total data set has been plotted. Each difference spectrum has been given a vertical offset of $2 \times 10^{15} \mathrm{ergs} \mathrm{s}^{-1} \mathrm{~cm}^{-2} \AA^{-1}$ for display purposes. The (vertical) flux scale is linear, and the zero point for each difference spectrum is indicated by a dotted line. The Julian date identification of each spectrum is given along the right of the panel.

During the first three years of monitoring the spectral resolution was slightly worse than during the fourth to sixth year of monitoring due to the detector change between the third and fourth year. This shows up in the form of typical "resolution residuals" in the [O III] lines during the good resolution years. These residuals do not signify errors in the flux calibration process, but they are due to the subtraction of two spectra with slightly different resolution.

Note that the profile variations in both $\mathrm{He}$ II and $\mathrm{H} \beta$ are generally slowly varying from one epoch to the next and do not follow a random pattern.

\subsubsection{The First Monitoring Year: 1989}

The $\mathrm{H} \beta$ profile during the first year, 1989 (Fig. 4a), starts off being slightly stronger than average at JD 7788 . Note how narrow this difference profile is.

During the first three epochs of the first year (JDs 7788, 7831,7837 ) the continuum flux decreased by about $20 \%$ (see Fig. 1). The $\mathrm{H} \beta$ line responds to this by decreasing its flux as well, but note that the blue side of the emission line responds faster than the red side; the red wing lags the blue wing. These variations take place in the very center of the line only; the far wings seem unaffected. The $\mathrm{H} \beta$ line reaches an absolute minimum at JD 7852, whereafter it gradually increases in flux again (see Fig. 1). Again, the blue 


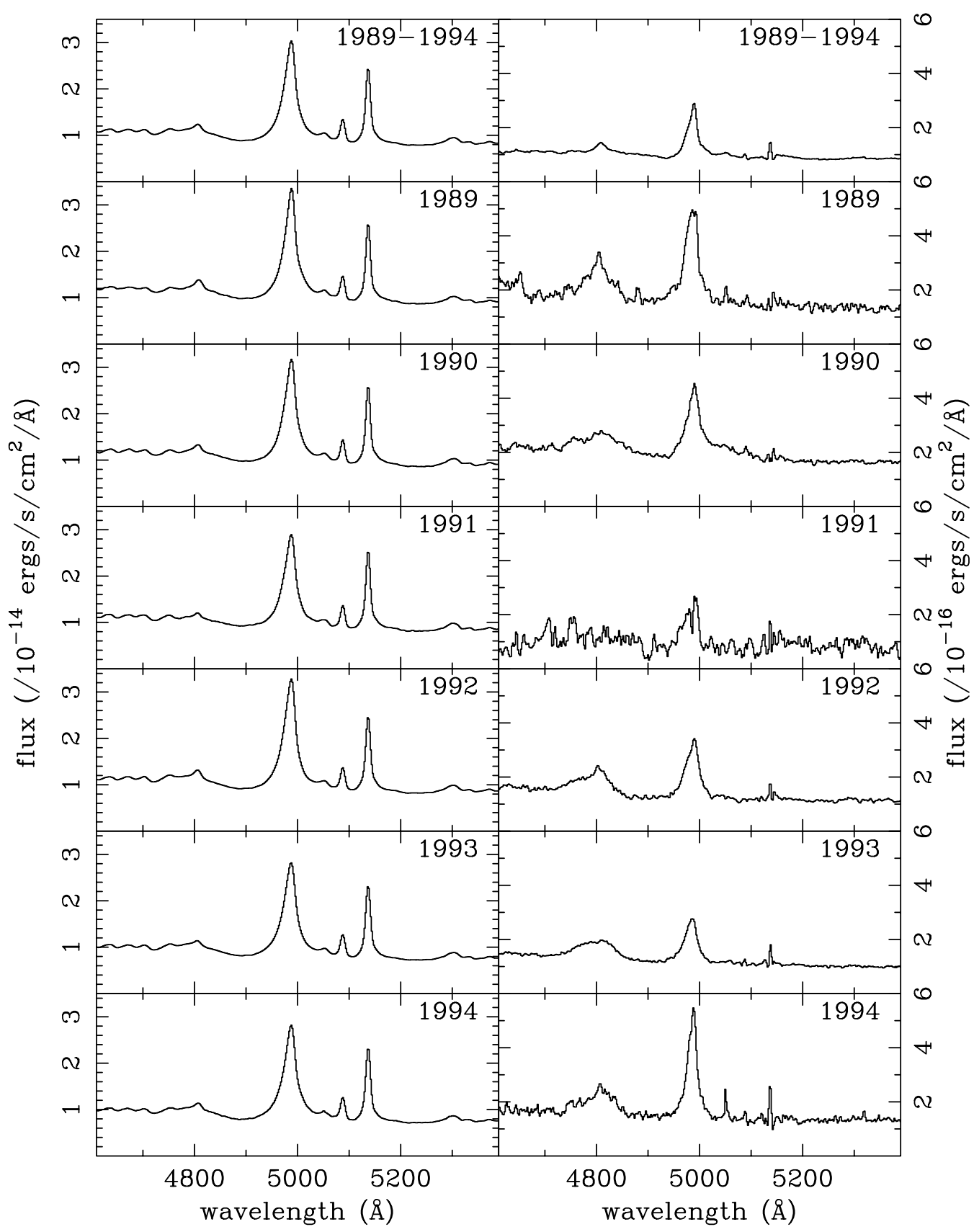

FIG. 3.-Average (left column) and root mean square spectra (right column) of the total data set (upper row) and the individual monitoring years

side appears to rise before the red wing in response to the simultaneous rise in the continuum flux.

The He II variations are weak during this first monitoring year, but a broad He II feature is discernible that varies in accordance with the blue side of the $\mathrm{H} \beta$ line, i.e., a fast response to the continuum variations.

\subsubsection{The Second Monitoring Year: 1990}

The first two observations of the second monitoring year, 1990 (Fig. 4b), were taken about 3 months before the rest, but the profile variations during this long period, which was a result of down time due to an electronics failure, seem to have been minimal. The largest change occurred in the continuum flux, which increased by about $20 \%$ during this time interval (see Fig. 1). At JDs 8187 and 8193, both the $\mathrm{H} \beta$ and the $\mathrm{He}$ II line respond to this increase in continuum flux by increasing their strength as well. Again, the variations in He II take place over a much broader range in radial velocity than the $\mathrm{H} \beta$ variations, which are confined to the core of the line.

The continuum flux starts a long decrease at JD 8193, while the $\mathrm{H} \beta$ line strength is still increasing. The $\mathrm{H} \beta$ response clearly lags the continuum variations. The $\mathrm{H} \beta$ flux is at its maximum around JD 8200-8207 and decreases thereafter. Note that during this increase and decrease of the $\mathrm{H} \beta$ profile, there is no indication that the blue side of the line responds before the red side, contrary to what was observed in the previous monitoring year.

The He II line is again observed to respond faster to the continuum variations than the $\mathrm{H} \beta$ line. It reaches a 

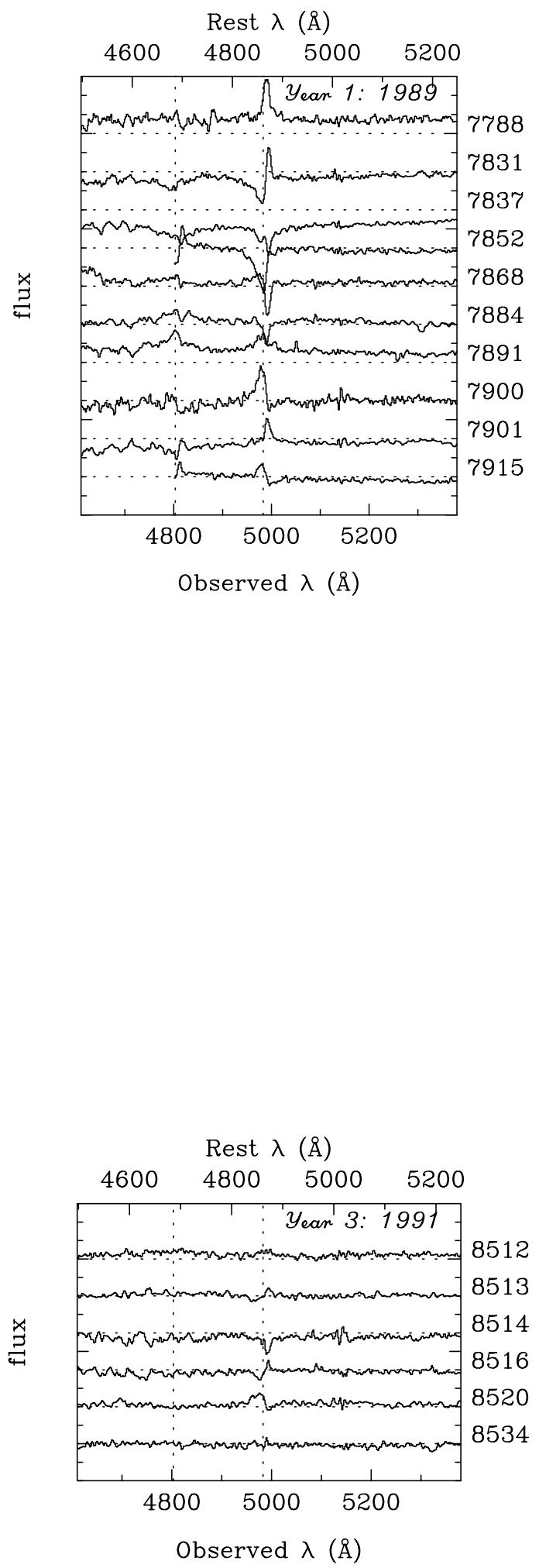
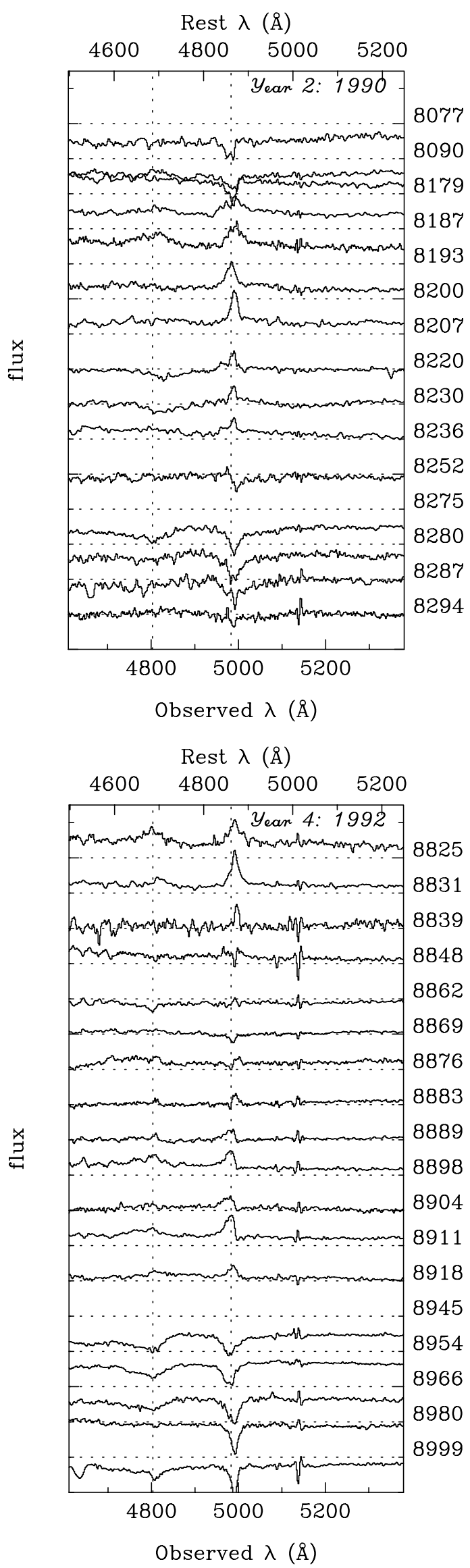

FIG. 4.-Difference spectra: spectrum taken at time $t_{i}$ minus the average spectrum of the total data set. The four least significant digits of the Julian Date of the observation are given for each difference spectrum to the right of the panel. In year six, the 9534 spectrum was included, owing to the fact that it is quite noisy. 

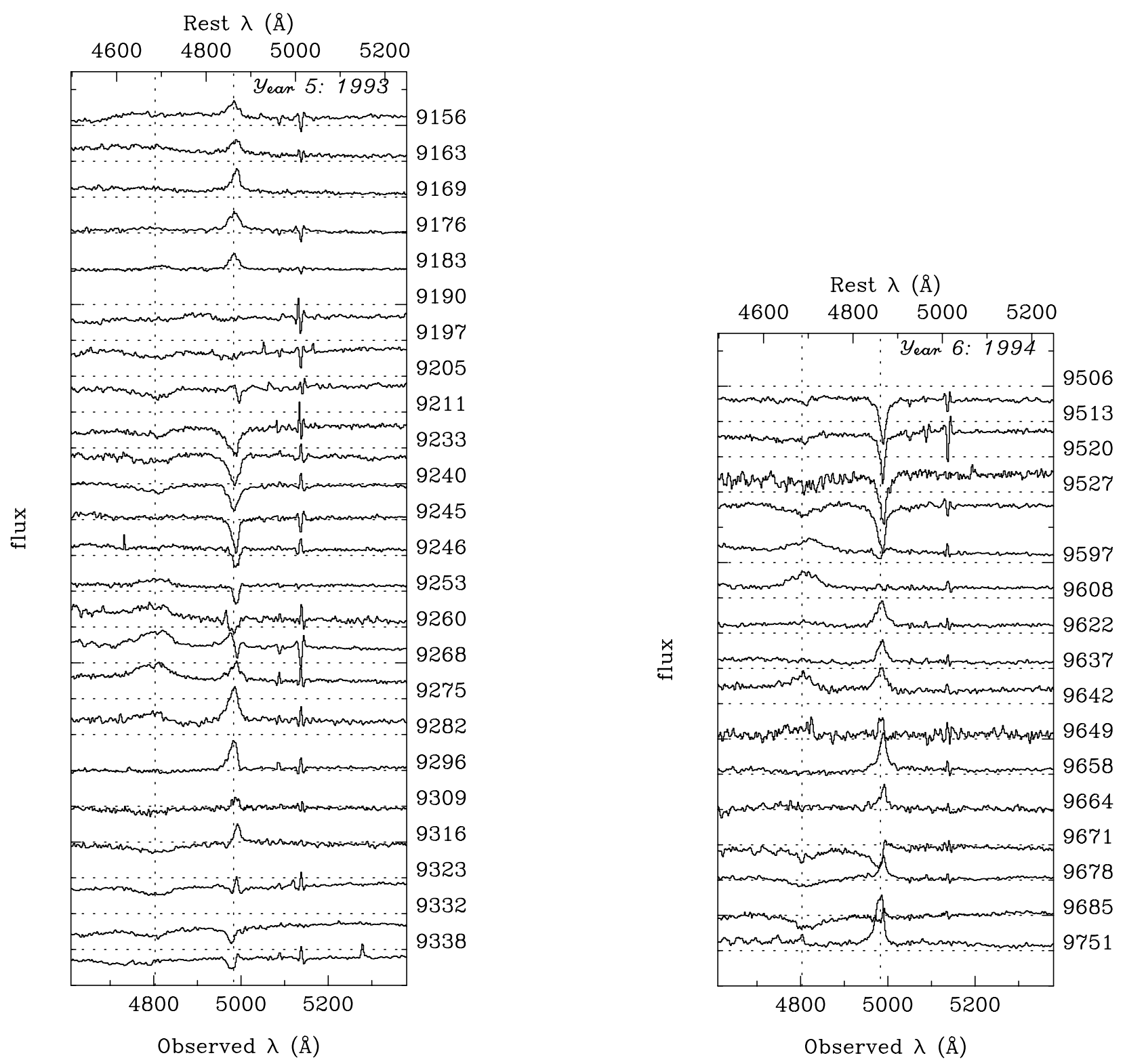

Fig. 4.-Continued

maximum flux the same epoch as the continuum (JD 8193) and then begins to decrease. The He II flux increases again at the end of the campaign, apparently simultaneously with the continuum flux variations, and well ahead of the $\mathrm{H} \beta$ flux variations.

\subsubsection{The Third Monitoring Year: 1991}

The third monitoring year, 1991 (Fig. 4c), is not well sampled and only six spectra were taken during a three week period. No significant profile variations are observed although the core of the $\mathrm{H} \beta$ line appears to have undergone small, narrow profile fluctuations.

\subsubsection{The Fourth Monitoring Year: 1992}

The fourth monitoring year, 1992 (Fig. 4d), is characterized by a general decrease in continuum and $\mathrm{H} \beta$ flux with superposed on that some short-term variability in the form of a small flux "outburst" around JD 8900 (see Fig. 1). This trend is easily followed in the difference spectra where the emission lines start out significantly stronger than average and end significantly weaker than average. The $\mathrm{H} \beta$ variations take place in the narrow core only, whereas the He II variations affect the outer wings as well.

The most remarkable feature during this year seems to be the very sharp boundary between the variable and nonvariable part of the $\mathrm{H} \beta$ line around JD 8898-8911. This feature will recur in later years as we will see shortly.

\subsubsection{The Fifth Monitoring Year: 1993}

The fifth monitoring year, 1993 (Fig. 4e), is the best sampled of all, with on average one spectrum taken each 7 or 8 days during a period of 6 months. It is clear that the profile variations form a smooth sequence in time. Fortunately, this year features large flux variations in both the continuum and the responding $\mathrm{H} \beta$ and $\mathrm{He}$ II lines (see Fig. 1 ), which are well sampled and have allowed the determination of the lags of $\sim 20$ days for $\mathrm{H} \beta$ and $\sim 0$ days for He II. 
Again we note that the $\mathrm{H} \beta$ variations are very strong in the narrow core only and very weak farther out in the wings, in contrast to the very broad He II profile variations.

At the beginning of the campaign the $\mathrm{H} \beta$ flux is stronger than average, it then drops to a minimum at JD 9240-9245, then rises to a maximum at JD 9282, whereafter it drops again toward the end of the monitoring campaign. Note that the He II line follows a similar pattern, albeit somewhat earlier in time. For example, the He II flux reaches a maximum flux at JD 9268.

Around JD 9260-9282, when the $\mathrm{H} \beta$ flux is at a maximum, it appears as if the blue side of the $\mathrm{H} \beta$ lines again responds earlier than the red side, just as it did in the first monitoring year, 1989 (§ 5.2.1). At the same time, this sharp feature in the profile, present in the previous monitoring year, 1992 (§ 5.2.4), shows up as well and appears to form a boundary between blue side and red side variability behavior.

\subsubsection{The Sixth Monitoring Year: 1994}

The sixth monitoring year, 1994 (Fig. 4f), is certainly the most dramatic one. The light curves (Fig. 1) present relative smooth behavior in time for the continuum flux, but rather erratic behavior for the $\mathrm{H} \beta$ emission-line flux. This is not expected if the $\mathrm{H} \beta$ light curve is supposed to be a convolution of the continuum light curve with a rather broad nonnegative transfer function.

The general trend of the $\mathrm{H} \beta$ flux is from a weak state in the beginning of the campaign to a strong state at the very end, but in between, significant variations are observed in both the strength and the shape of the $\mathrm{H} \beta$ emission line on timescales as short as the sampling interval.

During the first five epochs, nothing surprising occurs in the profiles. Then there is a relatively large time gap in the data of more than 2 months, between JD 9534 and 9597. Between these two epochs, the continuum flux increased more than $10 \%$ before reaching its maximum at JD 9608 . The He II emission line varies correspondingly and reaches its maximum at the same epoch as the continuum. Two weeks later, at JD 9622, the He II line already decreases in flux, as does the continuum, whereas the $\mathrm{H} \beta$ flux is lagging behind and is stronger on JD 9622 than on JD 9608. This behavior is shown in more detail in Figure 5, where the three difference spectra of JDs 9597, 9608, 9622, are shown in the left panel, whereas in the right panel the differences between the consecutive spectra of JD 9608 and 9597, and JD 9622 and 9608, are plotted. The right-hand panel is an especially clear illustration of the downward trend in He II and the upward trend in $\mathrm{H} \beta$.

During the period JDs $9597-9622$ the $\mathrm{H} \beta$ profile variations appear quite symmetrical. This time there is no sign of the blue side responding faster than the red side. This apparent line symmetry is not constant, however, and later that year, at JD 9671, the blue side of the $\mathrm{H} \beta$ line has plummeted in flux relative to the red side in a period of just 7 days. The He II flux also dropped significantly, whereas the change in continuum flux is less pronounced. Just 6 days later, JD 9678, the $\mathrm{H} \beta$ line gains in strength considerably, whereas the He II line and the continuum do not do so. Again, 1 week later, JD 9685, the $\mathrm{H} \beta$ flux dropped back to the average level, whereas the He II flux is below average.

This mysterious short-term behavior of the $\mathrm{H} \beta$ profile is not due to calibration errors, since it is not followed by a similar pattern in either the He II line or the continuum flux, nor are there any significant narrow-line residuals visible.

The last observation of the monitoring campaign is 2 months later than the second to last observation. The $\mathrm{H} \beta$ emission line is very strong, whereas the He II line is not.

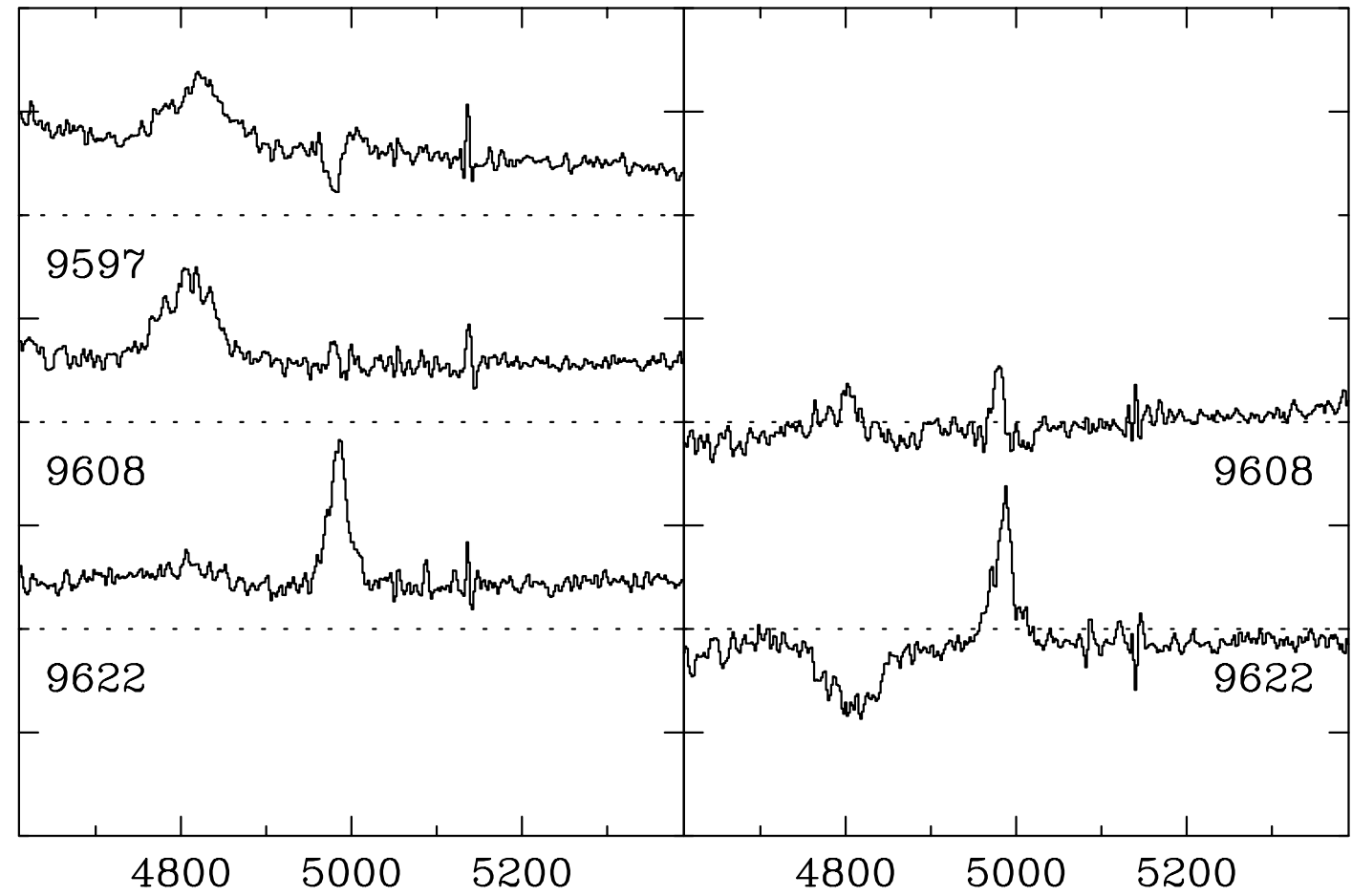

Fig. 5.-Detailed comparison of the responses of the H $\beta$ and He II emission lines around JD 9597-9622. The left panel shows the spectra from which the total average spectrum was subtracted [ $\mathrm{JD}(i)$ - average], and the right panel shows difference spectra produced by subtracting the spectrum of the previous epoch from the spectra observed at epochs 9608 and 9622 , respectively [JD(i) $-\mathrm{JD}(i-1)]$. 
This may be a response to an unobserved continuum outburst during the unobserved time interval just before the last observation, where the $\mathrm{H} \beta$ line still responds to this higher level of continuum flux, whereas the $\mathrm{He}$ II line is already past its maximum response.

\section{DISCUSSION AND SUMMARY}

We have presented an analysis of the flux and profile variability of the broad emission lines of the Seyfert 1 galaxy Mrk 335 over a period of six years. We find the $\mathrm{H} \beta$ variations lag those of the continuum by about 17 days, whereas those of the He II 24686 line respond to the continuum variations with a delay of less than 2 days. The $\mathrm{He}$ II response is indistinguishable from being instantaneous (lag $\Delta t=0$ days).

The average spectrum over the full data set shows that the $\mathrm{He}$ II line is about twice as broad as the $\mathrm{H} \beta$ line. The root mean square spectra provide evidence that the $\mathrm{H} \beta$ variations mainly occur in the core of the line, whereas the He II variations take place over the full width of the line.

Wanders \& Peterson (1996) found in the AGN NGC 5548 that the profile variations mainly take place in one central narrow core component and two (one red and one blue) broader wing components. In Mrk 335, we do not observe the equivalent of the wing components, but only the narrow core component. In terms of the anisotropic continuum model for the BLR (Wanders et al. 1995; Goad \& Wanders 1996), used by Wanders \& Peterson to explain the profile behavior of NGC 5548, Mrk 335 would have beamed continuum source that is directed straight toward the observer, with a small semiopening angle.
It is of great interest that the behavior of the line profiles varies considerably from year to year. This is exemplified by the fact that in some years the difference spectra show that the blue side of the $\mathrm{H} \beta$ line respond to changes in the continuum more rapidly than the red side of the line, whereas in other years there is no sign of this behavior, and the red and blue sides of the line vary in unison. A clear example of the blue side-first behavior is the first monitoring year with another, more marginal, case in the fifth monitoring year around JD 9260-9282. This trend of the blue side of the $\mathrm{H} \beta$ line responding more quickly than the red side has not been seen before in other monitored AGNs.

Crenshaw \& Blackwell (1990) and Done \& Krolik (1995) have described similar asymmetric line variations in NGC 5548, though they saw the red side of the line responding faster than the blue side, exactly the reverse of the Mrk 335 behavior. These authors interpreted this as evidence for a radial-infall component of the velocity field of the BLR gas. However, the opposite behavior detected in the present paper, which would suggest radially outflowing gas in the reverberation picture, leads us to believe that these contradictory results are probably the result of dynamical changes within the BLR (Perry, van Groningen, \& Wanders 1994) rather than being reverberation effects.

We are very grateful to the Director and staff of Lowell Observatory without whose support this work would not have been possible. We gratefully acknowledge support for this program by the National Science Foundation through grants AST-9117086 and AST-9420080 and by NASA through grant NAG5-2477.
Blandford, R. D., \& McKee, C. F. 1982, ApJ, 255, 419

Capriotti, E. R., Foltz, C. B., \& Peterson, B. M. 1982, ApJ, 261, 35

Clavel, J., et al. 1991, ApJ, 366, 64

Crenshaw, D. M., \& Blackwell, J. H., Jr. 1990, ApJ, 358, L37

Davidson, K., \& Netzer, H. 1979, Rev. Mod. Phys., 51, 715

Done, C., \& Krolik, J. H. 1996, ApJ, in press

Edelson, R. A., \& Krolik, J. H. 1988, ApJ, 333, 646

Edelson, R. A., Krolik, J. H., \& Pike, G. F. 1990, ApJ, 359, 86

Gaskell, C. M., \& Peterson, B. M. 1987, ApJS, 65, 1

Goad, M. R., \& Wanders, I. 1996, ApJ, 469, 113

Gondhalekar, P. G., Horne, K., \& Peterson, B. M., eds. 1994, ASP Conf. Ser. 69, Reverberation Mapping of the Broad-line Region in Active Galactic Nuclei (San Francisco: ASP)

Koratkar, A. P., \& Gaskell, C. M. 1991, ApJS, 75, 719

Netzer, H. 1991, in Saas-Fee Advanced Course 20, Active Galactic Nuclei, ed. T. J.-L. Courvoisier \& M. Mayor (Berlin: Springer), 57

\section{REFERENCES}

Penston, M. V. 1991, in Variability of Active Galactic Nuclei, ed. H. R. Miller \& P. J. Wiita (Cambridge: Cambridge Univ. Press), 343

Perry, J. J., van Groningen, E., \& Wanders, I. 1994, MNRAS, 271, 561 1993, PASP, 105, 247

Peterson, B. M., et al. 1991, ApJ, 368, 119

. 1993, ApJ, 402, 469

Peterson, B. M., Pogge, R. W., Wanders, I., Smith, S. M., \& Romanishin, W. J. 1995, PẢSP, 107, 579

Shuder, J. M. 1981, AJ, 86, 1595

van Groningen, E., \& Wanders, I. 1992, PASP, 104, 700

Wanders, I., \& Peterson, B. M. 1996, ApJ, 466, 174

Wanders, I., et al. 1995, ApJ, 453, L87

White, R. J., \& Peterson, B. M. 1994, PASP, 106, 879 